\title{
Impact of South African 501.V2 Variant on SARS-CoV-2 Spike Infectivity and Neutralization: A Structure-based Computational Assessment
}

\author{
Mary Hongying Cheng ${ }^{1}$, James M Krieger ${ }^{1}$, Burak Kaynak ${ }^{1}$, Moshe Arditi ${ }^{2}$ and Ivet Bahar ${ }^{1, *}$ \\ ${ }^{1}$ Department of Computational and Systems Biology, School of Medicine, University of Pittsburgh, Pittsburgh, PA 15213, \\ USA. ${ }^{2}$ Department of Pediatrics, Division of Pediatric Infectious Diseases and Immunology, and Biomedical Sciences, \\ Infectious and Immunologic Diseases Research Center, Cedars-Sinai Medical Center, Los Angeles, CA 90048, USA
}

*To whom correspondence should be addressed. bahar@pitt.edu

Associate Editor: XXXXXXX

Received on XXXXX; revised on XXXXX; accepted on XXXXX

\begin{abstract}
Motivation: The SARS-CoV-2 variants emerging from South Africa (501.V2) and the UK (B.1.1.7) necessitate rapid assessment of the effects of the corresponding amino acid substitutions in the spike (S) receptor-binding domain (RBD) of the variants on the interactions with the human ACE2 receptor and monoclonal antibodies (mAbs) reported earlier to neutralize the spike.

Results: Molecular modeling and simulations reveal that N501Y, shared by both variants, increases ACE2 binding affinity, and may impact the collective dynamics of the ACE2-RBD complex, occupying a central hinge site that modulates the overall dynamics of the complex. In contrast, the substitutions K417N and E484K in the South African variant 501.V2 would reduce the ACE2-binding affinity by abolishing two interfacial salt bridges that facilitate RBD binding to ACE2, K417(S)-D30(ACE2) and E484 (S)-K31(ACE2). These two mutations may thus be more than compensating the attractive effect induced by N501Y, overall resulting in an ACE2binding affinity comparable to that of the wildtype RBD. Further analysis of the impact of these mutations on the interactions with mAbs targeting the spike indicate that the substitutions K417N and E484K may also abolish the salt bridges between the spike and selected mAbs, such as REGN10933, BD23, H11_H4, and C105, thus reducing the binding affinity and effectiveness of these mAbs. Contact: bahar@pitt.edu

Supplementary information: Supplementary data are available at Bioinformatics online.
\end{abstract}

\section{Introduction}

The high transmissibility of SARS-CoV-2 and the lack of a robust pre-existing or acquired immunity by the hosts (Petersen, et al., 2020; Tortorici, et al., 2020) have caused COVID-19 cases to surge to more than 83 million over the world, resulting in over 1.8 million deaths by the end of 2020 . There is an urgent need to prevent infection through vaccination and/or develop effective therapies either by monoclonal antibody (mAb) treatments or development of antiviral compounds development to prevent and treat COVID-19 and associated multi-system inflammatory syndromes. Two vaccines produced by Pfizer/BioNTech and Moderna have already been authorized for emergency use in the US in December 2020, while the duration of the immune protection, their ability to protect populations across all age groups, as well as the capacity to produce sufficient doses of safe and effective vaccines remain to be determined (Krammer, 2020). Most importantly, even though the mutation rate of SARS-CoV-2 has been slower than that of SARS-CoV of 2003 and other SARSfamily coronaviruses, new SARS-CoV-2 variants emerged in recent weeks, which apparently increase transmissibility and induce immune escape (Andreano, et al., 2020; Kemp, et al., 2020; McCarthy, et al., 2020). Open genomic surveillance data sharing and collaborative online platforms allow us a real-time tracking of the emergence and spread of many virus lineages (Hadfield, et al., 2018; Shu and McCauley, 2017). As of December $11^{\text {th }}, 2020$, 246,534 SARS-CoV-2 sequences have been listed in the GIASID database (https:gisaid.org). Of note are the so-called UK B1.1.7 (Kemp, et al., 2020) and Southern African 501.V2 variants (Tegally, et al., 2020) which appear to be highly transmissible and have now been observed in multiple countries.

The SARS-CoV-2 spike (S) glycoprotein plays a major role in infectivity. $S$ is a trimer, each monomer being composed of two subunits, S1 and S2. The receptor binding domain (RBDs; residues 331-524) of the S1 subunit recognizes the human receptor 
bioRxiv preprint doi: https://doi.org/10.1101/2021.01.10.426143; this version posted January 11, 2021. The copyright holder for this preprint (which was not certified by peer review) is the author/funder, who has granted bioRxiv a license to display the preprint in perpetuity. It is made available under aCC-BY-NC-ND 4.0 International license.

angiotensin-converting enzyme 2 (ACE2); and the S2 trimer enables cell fusion and/or endocytosis following two cleavages by human proteases and ensuing massive conformational change. Not surprisingly, the majority of COVID-19 mAb therapies under investigation are designed to target the RBD, although other potential neutralizing epitopes have also been reported (Cao, et al., 2020; Cheng, et al., 2020; Chi, et al., 2020; Hansen, et al., 2020; Liu, et al., 2020; Pinto, et al., 2020; Renn, et al., 2020; Shi, et al., 2020; Yuan, et al., 2020). The spike is also the protein whose mRNA is used in the two emergency-authorized vaccines.

The UK variant contains up to eight substitutions/deletions in the S protein, including N501Y in the RBD; the South African variant 501.V2 has three (K417N, E484K and N501Y). Understanding the effect of these substitutions on the transmissibility and virulence of SARS-CoV- 2 as well as the effectiveness of mAbs known to neutralize the wild-type (wt) $\mathrm{S}$, is of broad and immediate interest.

In the present study, we assess the impact of the three RBDassociated amino acid substitutions in the South African variant 501.V2 spike on the interactions of the RBD with ACE2 and with neutralizing mAbs using a suite of molecular modeling and simulations. Our analysis shows that the mutations K417N and E484K act to counterbalance the high affinity of the spike imparted by the mutation N501Y (shared by both the UK and South African variants), suggesting that the South African variant may be less infectious and less transmissible than the UK variant. Furthermore, most of the structurally characterized mAbs (Table 1) including one of the two Regeneron mAbs used in combination (Hansen, et al., 2020) exhibit minimal 3D contacts or compensating interactions at the mutation sites, suggesting that their effectiveness will not be altered, and could even be increased in two cases, B38 (Wu, et al., 2020) and 2-4 (Liu, et al., 2020). In contrast, a weakening in the interactions with the variant is discerned in the cases of mAbs C105 (Barnes, et al., 2020), BD23 (Cao, et al., 2020), REGN10933 (Hansen, et al., 2020) and H11-H4 (Huo, et al., 2020), where the mutations K417N and/or E484K disrupt interfacial interactions that can be only partially compensated by conformational rearrangements. This computational analysis draws attention to the necessity of re-evaluating the neutralizing effect of selected mAbs vis-à-vis the potential ability of South African variant to escape immunotherapy.

\section{Methods}

In silico generation of structural models for SARS-CoV-2 S RBD variants complexed with ACE2 and mAbs. The structure of the complex formed by wt S RBD and ACE2 was obtained from the Protein Data Bank (PDB ID: 6LZG (Wang, et al., 2020)). To characterize the changes in interfacial interactions imparted by the amino acid replacements in the South African 501.V2 variant, we generated structural models using CHARMM-GUI for three mutant RBDs complexed with ACE2: (1) N501Y; (2) K417N and E484; and (3) K417N, E484K, and N501Y. The generated models were energetically minimized and further refined by molecular dynamics (MD) simulations with explicit solvent using HADDOCK 2. 2 (Van Zundert, et al., 2016). Similar modeling and simulations were performed for the complexes of the same variant with a
Table 1. MAbs whose interactions with the South African variant spike have been analyzed in the present study

\begin{tabular}{|c|c|c|}
\hline $\begin{array}{l}\mathrm{mAb} \text { (PDB } \\
\text { id) }\end{array}$ & $\begin{array}{l}\text { Epitope on SARS-CoV-2 S interface } \\
\text { with ACE }{ }^{b}\end{array}$ & References $^{c}$ \\
\hline $\begin{array}{l}\text { C105 } \\
(6 \times C N, \\
6 \times C M)\end{array}$ & $\begin{array}{l}\text { D405,T415, G416, K417, Y421, Y453, F456, } \\
\text { R457, K458, N460, Y473, A475, G476, F486, } \\
\text { N487, N501, G502, Y505 }\end{array}$ & $\begin{array}{l}\text { (Barnes, et al., } \\
\text { 2020) }\end{array}$ \\
\hline $\begin{array}{l}2-4 \\
\text { (6XEY) }\end{array}$ & $\begin{array}{l}\text { Y449, Y453, L455, F456, V483, E484, G485, } \\
\text { F486, Y489, F490, L492, Q493, S494 }\end{array}$ & $\begin{array}{l}\text { (Liu, et al., } \\
\text { 2020) }\end{array}$ \\
\hline $\begin{array}{l}\text { REGN10933 } \\
\text { (6XDG) }\end{array}$ & $\begin{array}{l}\text { K417, Y453, L455,F456, E484, G485, } \\
\text { F486,N487, C488, Y489, Q493 }\end{array}$ & $\begin{array}{l}\text { (Hansen, et } \\
\text { al., 2020) }\end{array}$ \\
\hline $\begin{array}{l}\text { REGN10987 } \\
\text { (6XDG) }\end{array}$ & $\begin{array}{l}\text { R346, N440, L441, K444, V445, G446, N448, } \\
\text { Y449, Q498 }\end{array}$ & $\begin{array}{l}\text { (Hansen, et } \\
\text { al., 2020) }\end{array}$ \\
\hline $\begin{array}{l}\text { B38 } \\
(7 \mathrm{BZ} 25)\end{array}$ & $\begin{array}{l}\text { R403, D405, Q409, T415, G416, K417, } \\
\text { D420, Y421, Y453, L455, F456, R457, K458, } \\
\text { N460, Y473, A475, G476, S477, F486, N487, } \\
\text { Y489, F490, Q493, Y495, G496, Q498, } \\
\text { T500, N501, G502 Y505 }\end{array}$ & $\begin{array}{l}(\mathrm{Wu}, \text { et al., } \\
2020)\end{array}$ \\
\hline 4A8 (7C2L) & $\begin{array}{l}\text { Y144, Y145, H146, K147, K150, W152, } \\
\text { H245, R246, S247, Y248, L249 }\end{array}$ & $\begin{array}{l}\text { (Chi, et al., } \\
\text { 2020) }\end{array}$ \\
\hline BD23 (7BYR) & $\begin{array}{l}\text { G446, Y449, E484, G485, F486, Y489, F490, } \\
\text { L492, Q493, S494, G496, Q498 N501, Y505, } \\
\text { N165 glycan }\end{array}$ & $\begin{array}{l}\text { (Cao, et al., } \\
2020)\end{array}$ \\
\hline EYGA (6ZDH) & $\begin{array}{l}\text { Y369, F374, S375, T376, F377, K378, C379, } \\
\text { Y380, G381, V382, S383, P384, T385, K386, } \\
\text { D389, L390, F392, P412, G413, D427, D428, } \\
\text { F429, T430 }\end{array}$ & $\begin{array}{l}\text { (Zhou, et al., } \\
\text { 2020) }\end{array}$ \\
\hline $\begin{array}{l}\text { H014 (7CAI, } \\
\text { 7CAC, 7CAB, } \\
\text { 7CAK,7CAH) }\end{array}$ & $\begin{array}{l}\text { Y369, A372,S373, F374, S375, T376, F377, } \\
\text { K378, C379, Y380, V382, S383, P384, T385, } \\
\text { D405, V407, R408, A411, P412, Q414, } \\
\text { N437, V503 }\end{array}$ & $\begin{array}{l}\text { (Lv, et al., } \\
2020)\end{array}$ \\
\hline $\begin{array}{l}\text { H11-H4 } \\
\text { (6ZBP, 6ZHD, } \\
\text { 6ZH9) }\end{array}$ & $\begin{array}{l}\text { Y449, N450, L455, F456, T470, G482, V483, } \\
\text { E484, Y489, F490, L492, Q493, S494, }\end{array}$ & $\begin{array}{l}\text { (Huo, et al., } \\
2020)\end{array}$ \\
\hline $\begin{array}{l}\text { CR3022 } \\
\text { (6ZLR, 6W7Y, } \\
\text { 6ZH9, 7A5R) }\end{array}$ & $\begin{array}{l}\text { Y369, N367, S375, T376, F377, K378, C379, } \\
\text { Y380, G381, V382, S383, P384, T385, K386, } \\
\text { L390, D428, F429, T430, F515, L517 }\end{array}$ & $\begin{array}{l}\text { (Huo, et al., } \\
2020)\end{array}$ \\
\hline ACE2 $^{\mathrm{a}}$ & $\begin{array}{l}\text { Epitope on SARS-CoV-2 S interface } \\
\text { with ACE2 }{ }^{b}\end{array}$ & Reference $^{c}$ \\
\hline $\begin{array}{l}\text { ACE2 } \\
\text { (6LZG) }\end{array}$ & $\begin{array}{l}\text { K417, G446, Y449, Y453, L455, F456, A475, } \\
\text { G476, E484, F486, N487, Y489, F490, Q493, } \\
\text { G496, Q498, T500, N501, G502, Y505 }\end{array}$ & $\begin{array}{l}\text { (Wang, et al., } \\
\text { 2020) }\end{array}$ \\
\hline
\end{tabular}

aProtein Data Bank ids of the structure resolved for S (or RBD) complexes with the indicated mAbs (top nine rows), and human receptor ACE2 (last row). Note that representative structures are shown among those in the PDB. Those $m A b s$ written in boldface contain mutated residues at their interface with $S$. ${ }^{b}$ Residues on $S$ protein making close $(<4.0 \AA$ atom-atom) contacts with the $m A b s$ or $A C 2$. South African variant mutation sites are in boldface.

'Reference for the structure used as template in modeling and simulations.

series of mAbs using as template the PDB structures listed in Table 1. Those resolved at low resolution (e.g. BD23) were also subjected to energy minimization prior to modeling and simulations.

Assessment of changes in binding energetics due to amino acid variations. For each RBD-ACE2 complex model, four energetically favorable conformations sampled during MD refinement were selected to estimate the changes of binding free energy due to amino acid substitutions, using PRODIGY (Xue, et al., 2016). The same approach was adopted for the RBD-mAb complexes.

Characterization of impact of mutations on the structural mechanics of the $S$ protein complexed with ACE2. The global dynamics of RBD-ACE2 complex was evaluated using the Gaussian network model (GNM) (Bahar, et al., 1997), as implemented in 
bioRxiv preprint doi: https://doi.org/10.1101/2021.01.10.426143; this version posted January 11, 2021. The copyright holder for this preprint (which was not certified by peer review) is the author/funder, who has granted bioRxiv a license to display the preprint in perpetuity. It is made available under aCC-BY-NC-ND 4.0 International license.

South African variant infectivity and neutralization

the DynOmics web server (Li, et al., 2017), and by essential site scanning analysis (ESSA) (Kaynak, et al., 2020). Both tools are accessible and described in detail in the open source ProDy API (Bakan, et al., 2014; Bakan, et al., 2011). GNM analysis was used to determine the role of the mutation sites in the global dynamics of the S protein (wt or variant) complexed with ACE2, using broadly established analytical methods (Bahar, et al., 2010). Mainly, we evaluated the conformational flexibility (or meansquare fluctuations (MSFs)) of residues in the most cooperative modes of motions and identified the most constrained regions (i.e. minima in these MSFs plotted as a function of residue index, called global mobility profiles). These regions serve as anchors or hinges; as such they play a key mechanical role and cannot easily adapt to substitutions (Haliloglu and Bahar, 2015). ESSA, on the other hand, determine the essential residues whose perturbation alters the frequency dispersion of global motions. Residuebased ESSA scores are evaluated based on three lowest frequency GNM modes. High scores residues usually occupy functional (e.g. orthosteric or allosteric) sites (Kaynak, et al., 2020).

\section{Results}

N501Y induces an increase in ACE2 binding affinity, and alters the structural mechanics of the RBD-ACE2 complex. The amino acid N501, mutated to Y501 in both the UK and South African variants, forms two hydrogen bonds with the residues $Y 41$ and K353 of ACE2, resulting in a stable complex with ACE2, with a binding affinity of $-12.1 \pm 0.2 \mathrm{kcal} / \mathrm{mol}$ (Fig. 1B). N501Y mutation further induces three new associations with ACE2: (i) a hydrogen bond with D38, (ii) a cation- $\pi$ interaction with $\mathrm{K} 353$, and a potentially $\pi$-stacking interaction with $Y 41$, resulting in an increase in ACE2-binding affinity to $-12.6 \pm 0.3 \mathrm{kcal} / \mathrm{mol}$. Note that the interaction partners $\mathrm{Y} 41$ and $\mathrm{K} 353$ are critical residues that have been reported to regulate ACE2-SARS binding: $\mathrm{K} 353 \mathrm{H}$ ( $A$ or $\mathrm{D}$ ) is known to abolish the interaction with the SARS-CoV S glycoprotein; and Y41A strongly inhibits interaction with the SARS-CoV S glycoprotein (Li, et al., 2005). Therefore, in both the UK and South African

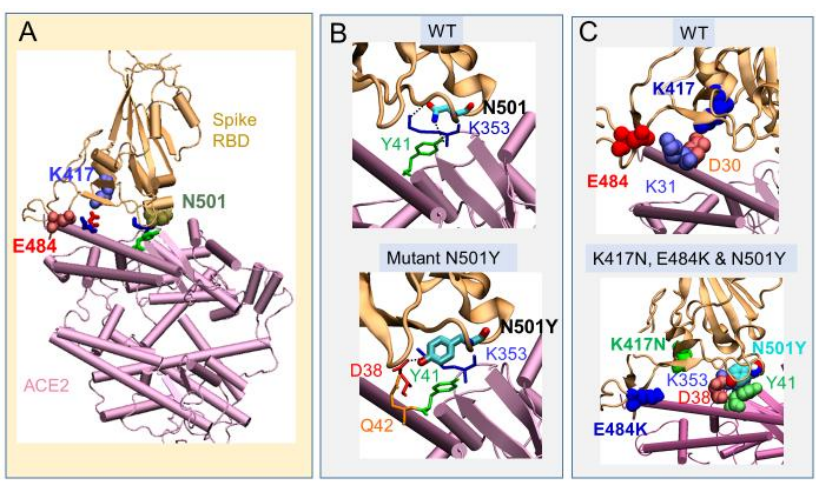

Fig 1. Change in interactions with ACE2 between wt RBD and Southern African variant 501.V2. (A) position of the three amino acids (K417, N501 and E584) at the interface with human ACE2 in the WT RBD; (B) comparison of the interactions of N501 (top) with those of the variant with single substitution N501Y (bottom). The latter enables a tighter contact resulting in higher binding affinity compared to WT. (C) Same as B for the triple mutant K417N, D501Y and E484K. Two salt bridges originally present in the wt (top) are broken (bottom), suggesting a compensating effect by the substitutions E484K and K417N, countering N501Y. variants, the substitution N501Y is expected to promote ACE2 binding and thereby induce an increase in transmissibility. This result is consistent with the results from deep mutational scanning experiments (Starr, et al., 2020) as well as a rigorous computational analysis (Fratev, 2020).

K417N and E484K mutations abolish two salt bridges otherwise formed between ACE2 and S RBD. K417 is specific to SARS-CoV$2 \mathrm{~S}$. The salt bridge between K417 and D30 from ACE2 assists in RBD-ACE2 binding (Wang, et al., 2020). In addition, an interfacial salt-bridge between S E484 and ACE2 K31 further stabilizes the complex (Fig. 1C top). Notably, the mutations (K417N and E484K) in the South African variant abolish these interfacial salt bridges reducing the binding affinity of the RBD to $-11.4 \pm 0.3 \mathrm{kcal} / \mathrm{mol}$ (in the absence of the $3^{\text {rd }}$ substitution N501Y). This decrease in affinity appears to counterbalance the increase imparted by $\mathrm{N} 501 \mathrm{Y}$, suggesting that the two additional mutations in the South African variant 501.V2 act to compensate the effect of N501Y. This compensating effect is not present in the UK variant.

Most of the mAbs reported to neutralize the spike show little sensitivity to the substitutions in the South African triple variant. We analyzed a set of eleven antibodies (Table 1, first column) whose structures complexed with the SARS-CoV-2 spike or RBD have been resolved. Fig. 2 displays their binding poses, generated by structurally aligning the spike or RBD regions of the corresponding S/RBD - mAb complex structures. Table 1 column 2 lists the residues that make interfacial contacts with the mAbs. Examination of the structures showed that the three mutation sites (K417N, E484K and N501Y) on the South African variant do not engage in direct contacts with five of the listed $m A b s$ : REGN10987, EY6A, HO14, 4A8, and CR3022 (Suppl Fig 1), suggesting that their substitutions would affect the effectiveness of these mAbs only minimally, if at all.

The remaining six $\mathrm{mAbs}$ are engaged in direct contacts with the variant spike at one or more of the three mutation sites. Among them, B38 and 2-4 tend to even exhibit an increased affinity to bind to the variant, compared to their affinity to bind the wt RBD. The corresponding interfacial contacts are illustrated in Suppl Fig 2A-B. Mainly, the substitution E484K allows for new favorable interactions with $\mathrm{mAb} 2-4$ residues $\mathrm{S} 54$ and N52; and the mutation N501Y leads to a hydrogen bond with mAb 2-4 T28, both in favor of tighter binding of 2-4 to the variant (Fig 2A right panel). Likewise, K417N creates a new hydrogen bond with B38 residue $\mathrm{Y33}$, and the network of interfacial interactions between RBD pair of residues N501 and Q498 and the mAb B38 pair S67 and $\mathrm{S} 30$ is maintained if not strengthened upon substitution of a
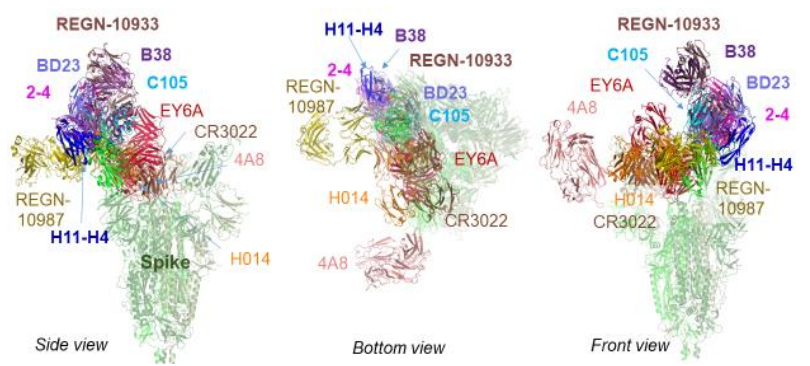

Figure 2. Interactions of the SARS-CoV-2 $S$ with mAbs. See Table 1 for the list of $m A$ bs and binding epitopes on the $S$ protein. 
bioRxiv preprint doi: https://doi.org/10.1101/2021.01.10.426143; this version posted January 11, 2021. The copyright holder for this preprint (which was not certified by peer review) is the author/funder, who has granted bioRxiv a license to display the preprint in perpetuity. It is made available under aCC-BY-NC-ND 4.0 International license.

tyrosine for N501. Quantitative assessment of binding affinities in these two cases show a change of $0.4-0.5 \mathrm{kcal} / \mathrm{mol}$ in favor of binding, which is small but robustly reproduced in four independent runs.

The mutations K417N and/or E484K in the South African variant decrease the ability of the mAbs C105, BD23, REGN10933, and H11_H4 to bind the RBD. C105 exhibits a decrease in binding affinity to the variant, compared to its affinity to bind the wt RBD. C105 depends on K417 to coordinate a polar network that supports its high affinity to bind the S RBD. K417 forms a salt bridge with the $\mathrm{C} 105$ residues E96/E99 which also engages the spike residue Y453 (see Fig 3A). This network is completely abolished by the substitution K417N (highlighted by the red circle in panels $\mathbf{A}$ and $\mathbf{B}$ of Fig $\mathbf{3}$ ). This significant loss in the attractive interaction with the mAb C105 is partially alleviated by a new favorable interaction between the $\mathrm{N} 417$ and $\mathrm{mAb} Y 52$, and a cation- $\pi$ interaction that Y501 makes with mAb K31. But the net effect is a reduction in binding affinity by $0.4 \mathrm{kcal} / \mathrm{mol}$.

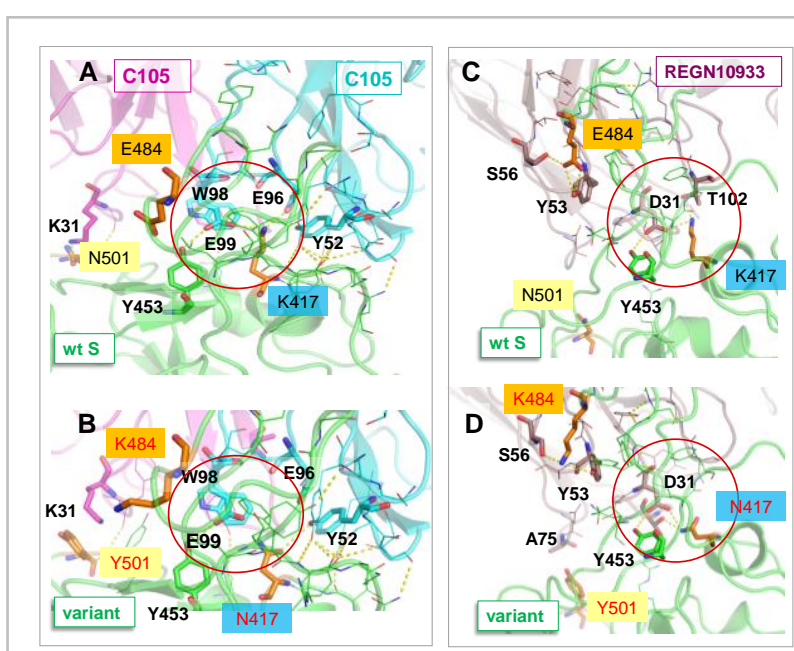

Figure 3: Disruption of salt bridges formed by K417 may weaken the association of C105 and REGN10933 with the South African variant 501.v2, compared to their interaction with the wt $S$. The panels compare the interactions of the mAbs C105 (A-B) and REGN10933 (C-D) with the wt RBD ( $A$ and $C$ ) and the South African mutant (B and D). A central salt bridge with C105 (K417-E99/E96) and another with REGN10933 (K417-D31) are lost due to the substitution K417N. A new cation- $\pi$ interaction with $\mathrm{K} 31$ is formed upon N501Y mutation in $\mathrm{D}$, which may compensate to restore the binding of REGN10933. N501Y makes no interfacial contacts, and E484K undergoes rearrangements to alleviate the effect of charge change. The net effect due to those changes in interfacial interactions is a weakening in binding affinity by $0.4 \pm 0.2 \mathrm{kcal} / \mathrm{mol}$.

The South African variant spike also appears to engage in a weaker association, compared to the wt $S$, with three other mAbs: BD23, H11_H4, and REGN10933. Fig 3C-D illustrates the change in interfacial interactions in the case of REGN10933, and Supplementary Fig $\mathbf{3 A}$ and $\mathbf{B}$ the results for BD23 and H11_H4, respectively. In the wt S- REGN10933 complex, K417 forms a salt bridge with mAb D31 also engaging T102, which in the mutant is replaced by weaker polar interactions. Likewise, E484 forms a salt bridge with BD23 R107 (Suppl Fig 3A middle), which, on the contrary, gives rise to repulsion between two positively charged groups upon the substitution E484K in the variant. A similar, even stronger effect is observed at the interface between $\mathrm{H} 11-4$ and the RBD (Supp Fig 3B) where the strong attractive interaction of wt E484 with H11-4 R52 turns into a strong repulsion between R52 and K484.

Overall, the two substitutions K417N and E484K in the South African variant impact salt bridges otherwise formed between the wt RBD and those four mAbs; and the accompanying structural rearrangements do not provide equally strong (new) associations. The net effect is a reduction in the binding affinity of these $\mathrm{mAbs}$ by 0.3 to $0.6 \mathrm{kcal} / \mathrm{mol}$. Although this difference is small, it is robustly reproduced in four independent runs for each complex, and it maps to more than 2 -fold decrease in the binding affinity of these antibodies, which warrants experimental inspection of dosage-dependent neutralization abilities of these mAbs.

N501 and K417 are essential residues that mediate the global dynamics of the RBD-ACE2 complex. Apart from interfacial interactions, it is of interest to examine the role of mutated residues in the structural dynamics of the complex formed with ACE2. GNM (Bahar, et al., 1997) analysis of the global dynamics of the RBD-ACE2 complex revealed that N501 plays a central mechanical role, participating in a hinge center (minimum in the global mobility profile) as illustrated in Fig 4A and D. Further analysis of essential sites by ESSA (Kaynak, et al., 2020) pointed to two of the residues mutated in the South African variant (K417 and $\mathrm{N} 501$; in red), and $\mathrm{ACE} 2$ two residues (Y41 and $\mathrm{K} 353$; in blue) that have been reported ( $\mathrm{Li}$, et al., 2005) to regulate the ACE2-spike binding (Fig 4B). It is interesting to note that several residues gain functional importance (emerge as peaks) upon complexation of the spike with ACE2 (Fig 4C), including K417 and N501 in RBD, Y41 and K353 in ACE2, in addition to D30 and K31 in ACE2 located at the interface. We also identified a pocket in the immediate vicinity of K417 and N501 at the RBD-ACE2 interface (Fig 4E) which is predicted by ESSA to potentially serve as an allosteric modulator binding site that might interfere with the conformational dynamics of the complex.

\section{Discussion}

SARS-CoV-2 has shown significant mutations in recent months, which raised the question whether any of the new variants would show increased transmissibility, potential to evade monoclonal antibody treatment or altered response to vaccines designed based on wt spike. The high mutation rate may in part be responsible for the zoonotic nature of these viruses and points to a clear risk of still-undetected additional members of the coronavirideae family of viruses making the jump from their traditional hosts to humans in the future.

To date there has been a relatively limited evidence of SARSCoV-2 mutations that have a significant functional effect on the virus or on the disease. In particular, significant attention has been given to the D614G mutation in the S protein, which emerged early in the pandemic, and might be associated with increased transmissibility (Korber, et al., 2020; Plante, et al., 2020; Volz, et al., 2020; Yurkovetskiy, et al., 2020), and with escape from monoclonal antibody (mAb) and polyclonal serum mediated neutralization (Thomson, et al., 2020). A recent extensive study of 184 recurrent mutations (including D614G) identified in 
bioRxiv preprint doi: https://doi.org/10.1101/2021.01.10.426143; this version posted January 11, 2021. The copyright holder for this preprint (which was not certified by peer review) is the author/funder, who has granted bioRxiv a license to display the preprint in perpetuity. It is made available under aCC-BY-NC-ND 4.0 International license.

South African variant infectivity and neutralization

SARS-CoV-2 population (dataset of 46,723 SARS-CoV-2 isolates, sourced July 30, 2020) showed, however, no evidence of in-

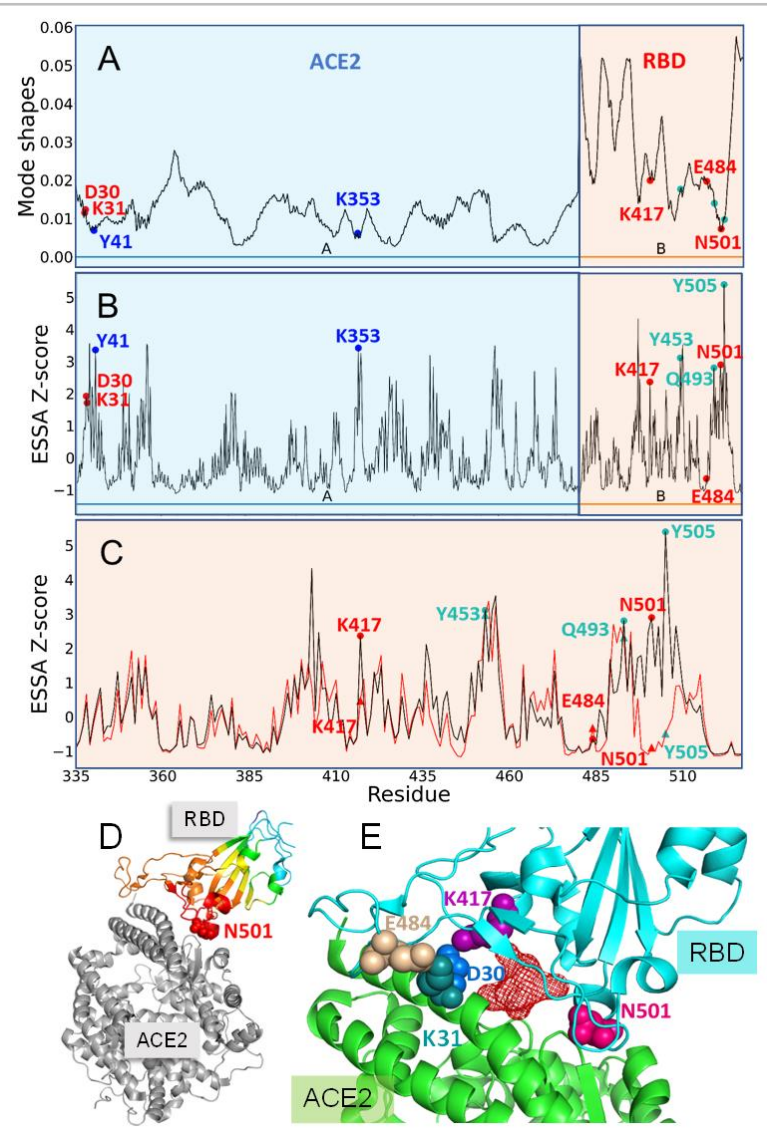

Figure 4. Role of mutation sites and other key sites in the structural dynamics of RBD complexed with ACE2. A. GNM-predicted global mobility profiles. Minima (sites participating in global hinge) include RBD N501 and ACE2 Y41 and E353. B Results from ESSA of RBD-ACE2 complex. The mutation sites K417 and N501, and associated ACE2 residues (D30 and K31, forming salt bridges with $\mathrm{K} 417$ and $\mathrm{E} 484$, respectively) are distinguished as peaks (essential residues). Three RBD residues implicated in the human-to-animal passage of the virus are labeled in cyan, which are also detected as essential residues. C. ESSA profile for isolated RBD (red curve), compared to its counterpart in the complex (black), shows that N501, K417, and Y505 acquire a significant mechanical role upon complexation. (D) Position of N501 in the RBD-ACE2 complex. RBD is color-coded by GNM mobility profile (from red; most constrained, to cyan, most mobile). (E) Closeup view of the interfacial regions displaying an allosteric pocket predicted by ESSA (red wire), and key residues in the vicinity.

creased transmissibility (van Dorp, et al., 2020).

Yet, in more recent months, the emergence of new UK and South African variants, which share a mutation N501Y at a critical (ACE2-binding) site, have once again raised concerns of high transmissibility. Furthermore, mutations in the $S$ protein could affect the efficacy of neutralizing Abs. Indeed, missense mutations that arose in MERS and SARS-CoV resulted in resistance to neutralizing antibodies for the original strains (Rockx, et al., 2010). One hypothesis for the emergence of immune escape variants, is that they arise through intra-host evolution in individuals with immunodeficiency and prolonged viral replication
(Avanzato, et al., 2020; Choi, et al., 2020). Indeed, the N501Y mutation is one of the several spike mutations that emerged in an immunocompromised individual in the US who had prolonged viral replication for over 20 weeks (Choi, et al., 2020).

In the present study, we focused on the South African 501.V2 variant and conducted a structure-based computational assessment of the effect of the corresponding substitutions N501Y, $\mathrm{K} 417 \mathrm{~N}$ and E484K in the S protein RBD on the interactions with ACE2 and neutralizing mAbs using the wealth of structural data accumulated to date for various complexes of the $S$ protein or its RBD with human ACE2 and many mAbs. While the full biological and clinical implications of the new variants in the UK and South Africa are yet to be determined, our study suggests that the affinity of the variant spike to recognize and bind to human ACE2, which is enhanced by the mutation N501Y, but weakened by the interactions engaging the other two mutation sites. As a result, current analysis suggests that despite the stronger binding to ACE2 caused by the substitution N501Y also noted in earlier experiments(Starr, et al., 2020) and computations (Fratev, 2020) for the point mutation, the South African 501.V2 variant that has undergone two additional mutations ( $\mathrm{K} 417 \mathrm{~N}$ and $\mathrm{E} 484 \mathrm{~K}$ ) is unlikely to exhibit an increased infectivity and likely transmissibility originating from an alteration of its interaction with human ACE2.

The effect of the mutations on existing mAbs is more heterogeneous. Here, we examined 11 mAbs (Table 1) reported to have neutralizing effects on wt virus, including two Abs used in the Regeneron combination $A b$ therapy that has been granted emergency use authorization. Five $\mathrm{mAb}$ binding epitopes (Epitope I to V) on the SARS-CoV-2 RBD have been identified, residues E484 and K417 contributing to Epitopes I and II (Xiang, et al., 2020). These two charged residues play an essential role in binding many mAbs by forming interfacial salt bridges with oppositely charged spike residues. The interactions between spike and four of the mAbs analyzed-C105, BD23, REGN10933 and H11-14, appear to be impacted due to disruption of salt bridges supported by E484 and K417. Specifically, K417 forms salt bridges with C105 E96/E99, and with REGN10933 D31; and E484 forms a salt bridge with BD23 R107 and with H11-14 R52. These attractive interactions are broken by the variant mutations, and in the case of the E484K mutation, may even be replaced by repulsions. Our analysis after considering local rearrangements that partially offset these adverse effects suggests that the RBD-binding probability of C105, BD23 and H11-14 may be reduced by a factor of two, while the effect on REGN10933 may be less than 2-fold.

On the contrary, for five of the examined mAbs, the mutations are predicted to exert only minimal effects on their interactions with RBD. It was also interesting to note that Fab 2-4 may have gained a 2-fold increase in its binding affinity, as E484K may promote an interfacial cation- $\pi$ interaction.

A recent mapping of RBD mutations that escape REGN-COV2 cocktail and Eli Lilly's LY-CoV016 antibodies (Starr, et al., 2020), using a deep mutational scanning method (Greaney, et al., 2020) revealed several sites whose point mutations mediate escape. K417 is indeed observed among those sites of 'strong escape' for REGN10933 (but not REGN10987) consistent with our study. Interestingly the mutation $\mathrm{K} 417 \mathrm{~N}$ is also the 'top' site of escape for LY-CoV016. The study further revealed that F486 is the strongest such site that escaped neutralization by REGN10933, and K444- 
bioRxiv preprint doi: https://doi.org/10.1101/2021.01.10.426143; this version posted January 11, 2021. The copyright holder for this preprint (which was not certified by peer review) is the author/funder, who has granted bioRxiv a license to display the preprint in perpetuity. It is made available under aCC-BY-NC-ND 4.0 International license.

G446 escaped neutralization by REGN10987. We note that these residues make direct contacts with the respective mAbs (Table 1). But interestingly the mutation (E406W) escaped the cocktail of both REGN antibodies even though it does not make direct contacts with either mAb in the resolved structure, inviting attention to the necessity of considering structural changes induced by such drastic substitutions, which may not be revealed by existing structures of the complex between the wt spike and mAbs.

Overall, our analysis suggests that a loss in mAb efficacy by the mutations may be alleviated by combination treatments consisting of mAbs that bind alternative, non-overlapping epitopes and act via different neutralization mechanisms to block virus mutational escape (Baum, et al., 2020; Hansen, et al., 2020; Marovich, et al., 2020; Xiang, et al., 2020) . A potential approach to minimize the impact of mAb escape mutations is indeed to develop additional mAbs with epitopes that are highly resistant to viral escape, such as those that include epitopes outside of the RBD and epitopes that are cross-reactive across SARS-CoV and SARS-CoV-2, indicating conserved epitopes with low tolerance for mutation (Pinto, et al., 2020; Wec, et al., 2020). We have recently described such a mAb (Cheng, et al., 2020) that binds the putative superantigenic-like motif of SARS-CoV-2 spike (Cheng, et al., 2020). That particular mAb, 6D3, presents the dual advantage of potentially blocking the S1/S2 cleavage site thus interfering with proteolytic cleavage that is essential for viral entry, in addition to targeting the superantigenic region that may contribute to multisystem inflammatory syndromes in in adults and in children and adolescents with severe COVID-19 (Cheng, et al., 2020).

In summary, we provide a computational analysis of the effect of the triple mutations in the South African variant on the infectivity and on the effectiveness of mAbs. The computational predictions provide testable hypotheses, such as that the optimal dosage in selected mAb treatments which may be affected by the mutations. Given the growing access to therapeutic mAbs via clinical trials and emergency use authorization, and the potential emergence of immune evasion mutations that maintain virulence or those that confer resistance to immunizations or therapies (Weisblum, et al., 2020), this type of in silico assisted genomic/molecular surveillance may provide feedback for accelerating the design of experimental studies in response to the pandemic.

\section{Acknowledgements}

We gratefully acknowledge support from the NIH awards 3RO1AI07272610 S1 (to MA) and P41GM103712 (to IB) and a MoISSI COVID-19 Seed Software Fellowship (to JK).

Conflict of Interest: none declared.

\section{References}

Andreano, E., et al. SARS-CoV-2 escape in vitro from a highly neutralizing COVID19 convalescent plasma. bioRxiv 2020:2020.2012.2028.424451.

Avanzato, V.A., et al. Case study: prolonged infectious SARS-CoV-2 shedding from an asymptomatic immunocompromised individual with cancer. Cell 2020;183(7):1901-1912.e1909.

Bahar, I., Atilgan, A.R. and Erman, B. Direct evaluation of thermal fluctuations in proteins using a single-parameter harmonic potential. Folding and Design 1997;2(3):173-181.
Bahar, I., et al. Global dynamics of proteins: bridging between structure and function. Annu Rev Biophys 2010;39:23-42.

Bakan, A., et al. Evol and ProDy for bridging protein sequence evolution and structural dynamics. Bioinformatics 2014;30(18):2681-2683.

Bakan, A., Meireles, L.M. and Bahar, I. ProDy: protein dynamics inferred from theory and experiments. Bioinformatics 2011;27(11):1575-1577.

Barnes, C.O., et al. Structures of human antibodies bound to SARS-CoV-2 spike reveal common epitopes and recurrent features of antibodies. Cell 2020;182:828842.

Baum, A., et al. Antibody cocktail to SARS-CoV-2 spike protein prevents rapid mutational escape seen with individual antibodies. Science 2020;369(6506):10141018

Cao, Y., et al. Potent neutralizing antibodies against SARS-CoV-2 identified by high-throughput single-cell sequencing of convalescent patients' B cells. Cell 2020;182(1):73-84.e16

Cheng, M.H., et al. A monoclonal antibody against staphylococcal enterotoxin B superantigen inhibits SARS-CoV-2 entry in vitro. bioRxiv 2020.

Cheng, M.H., et al. Superantigenic character of an insert unique to SARS-CoV-2 spike supported by skewed TCR repertoire in patients with hyperinflammation. Proc Natl Acad Sci USA 2020;117:25254-25262.

Chi, X., et al. A neutralizing human antibody binds to the N-terminal domain of the Spike protein of SARS-CoV-2. Science 2020;369(6504):650-655.

Choi, B., et al. Persistence and evolution of SARS-CoV-2 in an immunocompromised host. N Engl J Med 2020;383(23):2291-2293.

Fratev, F. The SARS-CoV-2 S1 spike protein mutation N501Y alters the protein interactions with both hACE2 and human derived antibody: A Free energy of perturbation study. BioRxiv 2020.

Greaney, A.J., et al. Complete Mapping of Mutations to the SARS-CoV-2 Spike Receptor-Binding Domain that Escape Antibody Recognition. Cell Host Microbe 2020 .

Hadfield, J., et al. Nextstrain: real-time tracking of pathogen evolution. Bioinformatics 2018;34(23):4121-4123.

Haliloglu, T. and Bahar, I. Adaptability of protein structures to enable functional interactions and evolutionary implications. Curr Opin Struct Biol 2015;35:17-23.

Hansen, J., et al. Studies in humanized mice and convalescent humans yield a SARSCoV-2 antibody cocktail. Science 2020;369(6506):1010-1014.

Huo, J., et al. Neutralizing nanobodies bind SARS-CoV-2 spike RBD and block interaction with ACE2. Nat Struct Mol Biol 2020;27(9):846-854.

Kaynak, B.T., Bahar, I. and Doruker, P. Essential site scanning analysis: A new approach for detecting sites that modulate the dispersion of protein global motions. Comput. Struct. Biotechnol. J. 2020;18:1577-1586.

Kemp, S., et al. Recurrent emergence and transmission of a SARS-CoV-2 Spike deletion $\Delta$ H69/V70. bioRxiv 2020:2020.2012.2014.422555.

Kemp, S.A., et al. Neutralising antibodies drive Spike mediated SARS-CoV-2 evasion. medRxiv 2020:2020.2012.2005.20241927.

Korber, B., et al. Tracking changes in SARS-CoV-2 Spike: evidence that D614G increases infectivity of the COVID-19 virus. Cell 2020;182(4):812-827.e819.

Krammer, F. SARS-CoV-2 vaccines in development. Nature 2020;586(7830):516527.

Li, H., et al. DynOmics: dynamics of structural proteome and beyond. Nucleic Acids Res 2017;45(W1):W374-w380.

$\mathrm{Li}, \mathrm{W}$, et al. Receptor and viral determinants of SARS-coronavirus adaptation to human ACE2. The EMBO Journal 2005;24(8):1634-1643.

Liu, L., et al. Potent neutralizing antibodies directed to multiple epitopes on SARSCoV-2 spike. Nature 2020;584:450-456.

Lv, Z., et al. Structural basis for neutralization of SARS-CoV-2 and SARS-CoV by a potent therapeutic antibody. Science 2020;369(6510):1505-1509.

Marovich, M., Mascola, J.R. and Cohen, M.S. Monoclonal Antibodies for Prevention and Treatment of COVID-19. JAMA 2020;324(2):131-132.

McCarthy, K.R., et al. Natural deletions in the SARS-CoV-2 spike glycoprotein drive antibody escape. bioRxiv 2020:2020.2011.2019.389916.

Petersen, E., et al. Comparing SARS-CoV-2 with SARS-CoV and influenza pandemics. The Lancet Infectious Diseases 2020;20(9):e238-e244.

Pinto, D., et al. Cross-neutralization of SARS-CoV-2 by a human monoclonal SARS-CoV antibody. Nature 2020;583(7815):290-295.

Plante, J.A., et al. Spike mutation D614G alters SARS-CoV-2 fitness. Nature 2020 
bioRxiv preprint doi: https://doi.org/10.1101/2021.01.10.426143; this version posted January 11, 2021. The copyright holder for this preprint (which was not certified by peer review) is the author/funder, who has granted bioRxiv a license to display the preprint in perpetuity. It is made available under aCC-BY-NC-ND 4.0 International license.

South African variant infectivity and neutralization

Renn, A., et al. Fruitful neutralizing antibody pipeline brings hope to defeat SARSCov-2. Trends Pharmacol. Sci. 2020;41:815-829.

Rockx, B., et al. Escape from human monoclonal antibody neutralization affects in vitro and in vivo fitness of severe acute respiratory syndrome coronavirus. J. Infect. Dis. 2010;201(6):946-955.

Shi, R., et al. A human neutralizing antibody targets the receptor-binding site of SARS-CoV-2. Nature 2020;584:120-124.

Shu, Y. and McCauley, J. GISAID: Global initiative on sharing all influenza data from vision to reality. Euro surveillance : bulletin Europeen sur les maladies transmissibles = European communicable disease bulletin 2017;22(13):30494.

Starr, T.N., et al. Prospective mapping of viral mutations that escape antibodies used to treat COVID-19. bioRxiv 2020.

Starr, T.N., et al. Deep Mutational Scanning of SARS-CoV-2 Receptor Binding Domain Reveals Constraints on Folding and ACE2 Binding. Cell 2020;182(5):12951310 e 1220 .

Tegally, H., et al. Emergence and rapid spread of a new severe acute respiratory syndrome-related coronavirus 2 (SARS-CoV-2) lineage with multiple spike mutations in South Africa. medRxiv 2020:2020.2012.2021.20248640.

Thomson, E.C., et al. The circulating SARS-CoV-2 spike variant N439K maintains fitness while evading antibody-mediated immunity. bioRxiv 2020:2020.2011.2004.355842.

Tortorici, M.A., et al. Ultrapotent human antibodies protect against SARS-CoV-2 challenge via multiple mechanisms. Science 2020:eabe3354.

van Dorp, L., et al. No evidence for increased transmissibility from recurrent mutations in SARS-CoV-2. Nat Commun 2020;11(1):5986.

Van Zundert, G., et al. The HADDOCK2. 2 web server: user-friendly integrative modeling of biomolecular complexes. J Mol Biol 2016;428(4):720-725.

Volz, E., et al. Evaluating the effects of SARS-CoV-2 Spike mutation D614G on transmissibility and pathogenicity. Cell 2020.

Wang, Q., et al. Structural and functional basis of SARS-CoV-2 entry by using human ACE2. Cell 2020;181(4):894-904 e899.

Wang, Y., Liu, M. and Gao, J. Enhanced receptor binding of SARS-CoV-2 through networks of hydrogen-bonding and hydrophobic interactions. Proc. Natl. Acad. Sci. U. S. A. 2020;117(25):13967-13974.

Wec, A.Z., et al. Broad neutralization of SARS-related viruses by human monoclonal antibodies. Science 2020;369(6504):731-736.

Weisblum, Y., et al. Escape from neutralizing antibodies by SARS-CoV-2 spike protein variants. Elife 2020;9.

Wu, Y., et al. A noncompeting pair of human neutralizing antibodies block COVID19 virus binding to its receptor ACE2. Science 2020;368(6496):1274-1278.

Xiang, Y., et al. Versatile and multivalent nanobodies efficiently neutralize SARSCoV-2. Science (New York, N.Y.) 2020;370(6523):1479-1484.

Xue, L.C., et al. PRODIGY: a web server for predicting the binding affinity of protein-protein complexes. Bioinformatics 2016;32(23):3676-3678.

Yuan, M., et al. A highly conserved cryptic epitope in the receptor binding domains of SARS-CoV-2 and SARS-CoV. Science 2020;368(6491):630-633.

Yurkovetskiy, L., et al. Structural and functional analysis of the D614G SARS-CoV2 Spike protein variant. Cell 2020;183(3):739-751.e738.

Zhou, D., et al. Structural basis for the neutralization of SARS-CoV-2 by an antibody from a convalescent patient. Nat. Struct. Mol. Biol 2020;27:950-958. 